\title{
Soluble cluster of differentiation 36 concentrations are not associated with cardiovascular risk factors in middle-aged subjects
}

\author{
MOHAMMAD J. ALKHATATBEH ${ }^{1}$, NEHAD M. AYOUB ${ }^{1}$, NIZAR M. MHAIDAT ${ }^{1}$, \\ NESREEN A. SAADEH ${ }^{2}$ and LISA F. LINCZ ${ }^{3}$ \\ ${ }^{1}$ Department of Clinical Pharmacy, Faculty of Pharmacy; ${ }^{2}$ Department of Internal Medicine, Faculty of Medicine, \\ Jordan University of Science and Technology, Irbid 22110, Jordan; ${ }^{3}$ Hunter Haematology Research Group, \\ Calvary Mater Newcastle Hospital, Waratah, NSW 2298, Australia
}

Received November 30, 2015; Accepted March 1, 2016

DOI: $10.3892 /$ br.2016.622

\begin{abstract}
Cluster of differentiation 36 (CD36) is involved in the development of atherosclerosis by enhancing macrophage endocytosis of oxidized low-density lipoproteins and foam cell formation. Soluble CD36 (sCD36) was found to be elevated in type 2 diabetic patients and possibly acted as a marker of insulin resistance and atherosclerosis. In young subjects, sCD36 was associated with cardiovascular risk factors including obesity and hypertriglyceridemia. The present study was conducted to further investigate the association between plasma sCD36 and cardiovascular risk factors among middle-aged patients with metabolic syndrome (MetS) and healthy controls. sCD36 concentrations were determined by enzyme-linked immunosorbent assays (ELISA) for 41 patients with MetS and 36 healthy controls. Data for other variables were obtained from patient medical records. sCD36 concentrations were relatively low compared to the majority of other studies and were not significantly different between the MetS group and controls $(\mathrm{P}=0.17)$. sCD36 was also not correlated with age, body mass index, glucose, lipid profile, serum electrolytes and blood counts. sCD36 was not significantly different between subjects with obesity, hyperglycemia, dyslipidemia, hypertension or cardiovascular disease, and those without these abnormalities $(\mathrm{P}>0.05)$. The inconsistency between results reported in the present study and other studies may be unique to the study population or be a result of the lack of a reliable standardized method for determining absolute $\mathrm{sCD} 36$
\end{abstract}

Correspondence to: Dr Mohammad J. Alkhatatbeh, Department of Clinical Pharmacy, Faculty of Pharmacy, Jordan University of Science and Technology, Irbid 22110, Jordan

E-mail: khatatbeh@just.edu.jo

Abbreviations: sCD36, soluble cluster of differentiation 36; MetS, metabolic syndrome; DM, diabetes mellitus; CVD, cardiovascular disease

Key words: metabolic syndrome, cluster of differentiation 36, cardiovascular risk, obesity, type 2 diabetes mellitus concentrations. However, further investigations are required to assess CD36 tissue expression in the study population and to assess the accuracy of various commercially available sCD36 ELISA kits. Thus, the availability of a standardized simple sCD36 ELISA that could be performed in any basic laboratory would be more favorable to the specialized flow cytometry methods that detect $\mathrm{CD} 6^{+}$microparticles if it was to be used as a biomarker.

\section{Introduction}

Cardiovascular disease (CVD) is the principal cause of fatalities among older adults worldwide (1). Advanced age, male gender and family history of CVD are considered as non-modifiable cardiovascular risk factors (2). Alternatively, modifiable risk factors for CVD include obesity, hypertension, hypercholesterolemia, hypertriglyceridemia, reduced high-density lipoprotein (HDL) cholesterol, diabetes mellitus (DM), smoking and a sedentary lifestyle (3). To best identify the subjects at an increased risk of CVD, the term 'Metabolic Syndrome' (MetS) emerged to describe a cluster of the modifiable risk factors that they can predict the occurrence of CVD when they exist together (4). However, subjects are diagnosed with MetS when they fulfill certain criteria that were defined by several health organizations (5-8).

Certain subjects may develop CVD without having numerous cardiovascular risk factors (9). Khot et al (10) have shown that at least one of the four traditional risk factors (smoking, DM, hyperlipidemia and hypertension) was found in $\sim 80 \%$ of their study population with CVD, suggesting that the development of CVD does not necessarily require the presence of a cluster of cardiovascular risk factors. Therefore, the prediction of CVD should not be restricted to the presence of cardiovascular risk factors, but it should also be based on measuring cardiovascular-specific risk biomarkers. Thus, identifying new biomarkers for CVD will improve the selection of subjects at increased risk of CVD for preventive strategies (9). Examples on current biomarkers for CVD were reviewed by Montgomery and Brown (11) and van Holten et al (12). These studies suggest that the biomarkers associated with lipids (such as HDL-cholesterol, triglycerides, cholesterol, apolipoprotein B and others) have 
stronger predictive potential for CVD compared to other biomarkers (12).

The initial process that leads to atherosclerosis, the hallmark of CVD, is foam cell formation (13). Foam cells are fat-laden macrophages that are produced by macrophage endocytosis of oxidized low-density lipoproteins (ox-LDLs) (13). This process is mediated by the macrophage transmembrane scavenger receptor cluster of differentiation 36 (CD36) (14). CD36 is not only implicated in ox-LDL endocytosis, but is also involved in triggering further signaling cascades that produce atherosclerotic inflammatory responses and is involved in regulating fat metabolism $(15,16)$. Notably, a circulating form of the transmembrane receptor CD36, termed soluble CD36 (sCD36), has been discovered in human plasma and was found to be associated with risk factors of atherosclerosis in people with type $2 \mathrm{DM}$, including insulin resistance and glycemic control (14). Thus, sCD36 was proposed to represent a marker of the MetS and a possible surrogate marker of atherosclerosis (14). Further studies have shown that plasma levels of sCD36 were elevated in obese type 2 diabetic patients compared to controls (14,17). Plasma sCD36 was also elevated and inversely correlated with measures of insulin sensitivity in patients with polycystic ovary syndrome (18). Furthermore, plasma sCD36 was increased in patients with symptomatic atherosclerotic carotid plaques and further associated with plaque instability (19).

In the present study, we hypothesize that plasma sCD36 may correlate with findings of the MetS among type 2 diabetic patients and may associate with an increased CVD risk among those patients. The aim was to validate the use of a simple, inexpensive, commercially available test for sCD36 as a biomarker of MetS and risk factors of CVD. To the best of our knowledge, this is the first study of absolute plasma sCD36 concentrations among middle-aged type 2 diabetic patients from Jordan.

\section{Patients and methods}

Study design. Patients with confirmed diagnosis of type 2 DM ( $n=110)$ were recruited from the diabetic clinic at King Abdullah University Hospital (KAUH; Ar Ramtha, Jordan). From this cohort, a sub-population $(n=41)$ who met the criteria of the MetS according to the International Diabetes Federation (IDF) definition was included in this study. Accordingly, diagnosis of MetS was based on having central obesity [waist circumference $\geq 94 \mathrm{~cm}$ for males and $\geq 80 \mathrm{~cm}$ for females or a body mass index $\left.(\mathrm{BMI})>30 \mathrm{~kg} / \mathrm{m}^{2}\right]$ plus any two of the followings: Systolic blood pressure $\geq 130 \mathrm{mmHg}$ or diastolic blood pressure $\geq 85 \mathrm{mmHg}$, or on treatment for hypertension, elevated triglyceride level ( $\geq 1.7 \mathrm{mmol} / \mathrm{l})$, or on treatment for this abnormality, reduced HDL-cholesterol $(<1.04 \mathrm{mmol} / \mathrm{l}$ for males or $<1.29 \mathrm{mmol} / \mathrm{l}$ for females), or on treatment for this lipid abnormality an increased fasting blood glucose (FBG) $\geq 5.6 \mathrm{mmol} / 1$, or diagnosed with type $2 \mathrm{DM}$ (8). All the patients were on medications for controlling blood glucose levels and for treatment of MetS abnormalities. The study sample was age- and gender-matched to healthy volunteers $(n=36)$ recruited from people who came to KAUH as visitors or for other purposes. All the subjects were recruited following obtaining informed consent and ethical approval by the
Institutional Research Board at KAUH and Jordan University of Science and Technology (reference no. 20140019).

Data collection and anthropometric measurements. Body weight $(\mathrm{kg})$, height $(\mathrm{cm})$ and waist circumference $(\mathrm{cm})$ were measured for all subjects as previously described (20). BMI was determined for all subjects according to the formula: BMI = body weight $(\mathrm{kg}) /$ height $\left(\mathrm{m}^{2}\right)$. The diagnosis of type 2 DM was made by the physicians according to the guidelines of the American Diabetes Association (21). Patients were considered hypertensive based on their medical history or receiving antihypertensive medications. For those who were not on antihypertensive medications, systolic and diastolic blood pressures were measured twice with a 10-min rest in between using a standardized mercury sphygmomanometer. The patient medical history including medications and other illnesses were obtained from their medical records. Patients were recruited between March and July 2014.

Blood sampling and processing. For plasma sCD36 determination, fasting venous blood samples were collected into $3.2 \%$ sodium citrate Vacuette tubes (Greiner Bio-One $\mathrm{GmbH}$, Kremsmünster, Austria). Plasma samples were prepared by double centrifugations at 3,000 x g for $15 \mathrm{~min}$ each at room temperature within $2 \mathrm{~h}$ of blood collection (16). Plasma aliquots were stored at $-80^{\circ} \mathrm{C}$ and were thawed at $37^{\circ} \mathrm{C}$ immediately before analysis. Other appropriate venous blood samples were also collected at the same time of the first sample in the KAUH biochemistry lab to determine levels of FBG, glycated hemoglobin (HgAlc), lipid profile, white blood cells (WBCs) and red blood cells (RBCs) counts, hemoglobin (Hgb), platelets, sodium, potassium, urea and creatinine serum levels.

Laboratory assays. FBG, HgA1c, lipid profile, WBCs, RBCs, $\mathrm{Hgb}$, platelets, sodium, potassium, urea and creatinine were determined by methods routinely used at KAUH biochemistry laboratory. Results for these variables were subsequently obtained from patient medical records or directly retrieved from the laboratory database for healthy controls that did not have medical records.

Plasma sCD36 level determination. Plasma sCD36 concentrations were measured by enzyme-linked immunosorbent assays (ELISA) obtained from (Aviscera Bioscience, Santa Clara, CA, USA) and according to the manufacturer's protocol. All the plasma samples were initially analyzed in duplicates using the human sCD36 ELISA kit (SK00196-02), which has a detection range of (1.95-250 $\mathrm{ng} / \mathrm{ml})$. Plasma samples were reanalyzed using a more sensitive human sCD36 ELISA kit (SK00196-06) with a detection range of (312.5-20,000 pg/ml). Intra-assay coefficient of variation was $<6 \%$ and inter-assay coefficient of variation was $<9 \%$.

Statistical analysis. Statistical analysis was performed using SPSS software (SPSS, Inc., Chicago, IL, USA) version 18. Differences between qualitative variables were determined using $\chi^{2}$ test. Continuous variables are expressed as mean \pm standard deviation or median (25-75th percentiles) where appropriate. Variables that were not normally distributed were log-transformed prior to analysis. Student's 
Table I. General, anthropometric and biochemical characteristics of the study groups.

\begin{tabular}{|c|c|c|c|}
\hline Variables & Healthy controls, $n=36$ & Metabolic syndrome, $n=41$ & P-value ${ }^{a}$ \\
\hline Gender, n (male/female) & $16 / 20$ & $18 / 23$ & $1.000^{\mathrm{b}}$ \\
\hline Age, years & $53.3 \pm 9.8$ & $54.3 \pm 9.7$ & 0.640 \\
\hline Waist circumference, $\mathrm{cm}$ & $90.9 \pm 9.9$ & $97.9 \pm 5.9$ & $<0.001$ \\
\hline $\mathrm{BMI}, \mathrm{kg} / \mathrm{m}^{2}$ & $28.3 \pm 4.7$ & $32.5 \pm 3$ & $<0.001$ \\
\hline $\mathrm{FBG}, \mathrm{mmol} / \mathrm{l}$ & $5.1 \pm 0.6$ & $9.4 \pm 3.4$ & $<0.001$ \\
\hline $\mathrm{HbA} 1 \mathrm{c}, \%$ & N/A & $8.4 \pm 1.9$ & N/A \\
\hline Duration of type $2 \mathrm{DM}$, years & N/A & $10.13 \pm 7.71$ & N/A \\
\hline \multicolumn{4}{|l|}{ Lipid profile, mmol/l } \\
\hline HDL-cholesterol & $1.10 \pm 0.26$ & $1.00 \pm 0.23$ & 0.070 \\
\hline LDL-cholesterol & $2.99 \pm 0.48$ & $2.86 \pm 0.90$ & 0.459 \\
\hline Total cholesterol & $4.4 \pm 0.6$ & $4.3 \pm 1.0$ & 0.583 \\
\hline Triglycerides & $1.56 \pm 0.32$ & $2.15 \pm 1.05$ & 0.002 \\
\hline \multicolumn{4}{|l|}{ Serum electrolytes, $\mathrm{mmol} / \mathrm{l}$} \\
\hline Sodium & $140.6 \pm 1.4$ & $139.1 \pm 3.0$ & 0.007 \\
\hline Potassium & $4.23 \pm 0.41$ & $4.57 \pm 0.55$ & 0.003 \\
\hline Urea & $4.7 \pm 1.1$ & $6.3 \pm 2.7$ & 0.001 \\
\hline Creatinine & $66.8 \pm 13.8$ & $81.1 \pm 35.5$ & 0.026 \\
\hline \multicolumn{4}{|l|}{ Blood counts } \\
\hline WBCs, x $10^{9} / 1$ & $6.94 \pm 1.63$ & $7.18 \pm 1.43$ & 0.504 \\
\hline RBCs, x $10^{9} / 1$ & $4.87 \pm 0.70$ & $4.75 \pm 0.52$ & 0.404 \\
\hline Platelets, x $10^{9} / 1$ & $279 \pm 65$ & $292 \pm 60$ & 0.378 \\
\hline $\mathrm{Hgb}, \mathrm{g} / \mathrm{l}$ & $13.28 \pm 1.42$ & $12.81 \pm 1.22$ & 0.123 \\
\hline Ln sCD36, pg/ml & $7.92 \pm 0.85$ & $8.18 \pm 0.83$ & 0.174 \\
\hline
\end{tabular}

${ }^{a}$ Student's t-test, ${ }^{\mathrm{b}} \chi^{2}$ test. BMI, body mass index; FBG, fasting blood glucose; HbA1c, glycated hemoglobin; DM, diabetes mellitus; HDL, high-density lipoprotein; LDL, low-density lipoprotein; WBCs, white blood cells; RBCs, red blood cells; Hgb, hemoglobin; sCD36, soluble cluster of differentiation 36; N/A, not available; Ln, natural logarithm.

t-test was used to assess differences in mean levels of normally distributed continuous variables. Correlations between sCD36 levels and other continuous variables were determined by the Pearson Product-Moment test. Multiple linear regression analysis was performed to detect the independent predictors of plasma sCD36 concentrations. All P-values were two-sided and $\mathrm{P}<0.05$ was considered to indicate a statistically significant difference.

\section{Results}

General, anthropometric and biochemical characteristics. Table I illustrates differences in the general, anthropometric and biochemical characteristics between patients with MetS $(n=41)$ and their matched healthy controls $(n=36)$. Measurements of waist circumference, BMI, FBG and triglycerides were significantly higher in patients with MetS compared to the controls $(\mathrm{P}<0.001)$. Additionally, measurements that reveal kidney function including potassium, urea and creatinine were significantly higher whereas sodium was significantly lower in patients with MetS compared to controls $(\mathrm{P}<0.03)$. Other measurements were not significantly different between patients with MetS and controls $(\mathrm{P}>0.05)$.
Measurement of plasma sCD36 levels in patients and controls. Plasma levels of sCD36 in the MetS and control groups were undetectable by the human sCD36 ELISA kit, which has a detection range of $(1.95-250 \mathrm{ng} / \mathrm{ml})$. Plasma levels of sCD36 of the same subjects were detected when the samples were reanalyzed using a more sensitive human sCD36 ELISA kit with a detection range of (312.5-20,000 pg/ml). As shown in Table I, results from this analysis did not show any significant difference in SCD36 concentrations between patients with the MetS and controls $(\mathrm{P}=0.17)$. Furthermore, sCD36 concentrations were found to be extremely low in the MetS group [2,991 (2,006-7,199 pg/ml)] and the control group [3,044 (1,625-4,843 pg/ml)].

Correlations between sCD36 and other variables. Plasma sCD36 levels in all the subjects and in the MetS and control groups were not significantly correlated with age, BMI, waist circumference, FBG, HDL-cholesterol, LDL-cholesterol, total cholesterol, triglycerides, sodium, potassium, urea, creatinine, WBC count, RBC count, Hgb and platelet count. Plasma sCD36 levels in the MetS group were also not significantly correlated with $\mathrm{HgAlc}$ and duration of type $2 \mathrm{DM}(\mathrm{P}>0.05)$ (Table II). Multiple linear regression analysis did not show any 
Table II. Correlation between soluble cluster of differentiation 36 concentrations and the other variables.

\begin{tabular}{|c|c|c|c|c|c|c|}
\hline \multirow[b]{2}{*}{ Variables } & \multicolumn{2}{|c|}{ All subjects, $n=77$} & \multicolumn{2}{|c|}{ Healthy controls, $n=36$} & \multicolumn{2}{|c|}{$\begin{array}{l}\text { Metabolic syndrome, } \\
\qquad \mathrm{n}=41\end{array}$} \\
\hline & $\mathrm{r}$ & P-value & $\mathrm{r}$ & P-value & $\mathrm{r}$ & P-value \\
\hline Age & -0.04 & 0.72 & 0.13 & 0.47 & -0.21 & 0.19 \\
\hline BMI & 0.09 & 0.43 & 0.12 & 0.50 & -0.12 & 0.46 \\
\hline Waist circumference & 0.07 & 0.56 & 0.03 & 0.85 & -0.03 & 0.83 \\
\hline FBG & 0.17 & 0.14 & 0.12 & 0.48 & 0.11 & 0.51 \\
\hline HDL-cholesterol & -0.17 & 0.15 & -0.14 & 0.43 & -0.14 & 0.39 \\
\hline LDL-cholesterol & -0.08 & 0.52 & 0.21 & 0.23 & -0.20 & 0.22 \\
\hline Total cholesterol & -0.11 & 0.32 & 0.08 & 0.66 & -0.21 & 0.19 \\
\hline Triglycerides & -0.01 & 0.93 & -0.32 & 0.06 & -0.02 & 0.92 \\
\hline Sodium & 0.03 & 0.79 & 0.08 & 0.64 & 0.09 & 0.58 \\
\hline Potassium & -0.09 & 0.44 & -0.02 & 0.90 & -0.24 & 0.13 \\
\hline Urea & 0.03 & 0.77 & 0.06 & 0.72 & -0.06 & 0.72 \\
\hline Creatinine & -0.06 & 0.60 & -0.01 & 0.97 & -0.15 & 0.34 \\
\hline WBCs & 0.10 & 0.40 & 0.03 & 0.86 & 0.14 & 0.37 \\
\hline $\mathrm{RBCs}$ & 0.04 & 0.73 & -0.01 & 0.97 & 0.13 & 0.41 \\
\hline Platelets & 0.08 & 0.47 & 0.01 & 0.93 & 0.12 & 0.45 \\
\hline $\mathrm{Hgb}$ & -0.04 & 0.70 & -0.03 & 0.86 & -0.002 & 0.99 \\
\hline $\mathrm{HgA} 1 \mathrm{c}$ & $\mathrm{N} / \mathrm{A}$ & N/A & N/A & N/A & 0.03 & 0.84 \\
\hline DM duration & N/A & N/A & N/A & N/A & -0.02 & 0.89 \\
\hline
\end{tabular}

r, Pearson product-moment correlation coefficient; BMI, body mass index; FBG, fasting blood glucose; HDL, high-density lipoprotein; LDL, low-density lipoprotein; WBCs, white blood cells; RBCs, red blood cells; Hgb, hemoglobin; HbA1c, glycated hemoglobin; DM, diabetes mellitus.

independent predictor of plasma sCD36 concentrations among all mentioned variables (data not shown).

Plasma sCD36 levels according to metabolic-associated abnormalities. When the entire cohort was stratified based on the presence/absence of specific individual metabolic variables (Table III), plasma sCD36 levels were not significantly different in subjects with obesity, fasting hyperglycemia, hypercholesterolemia, low HDL-cholesterol, high LDL-cholesterol, hypertriglyceridemia, hypertension and CVD compared to those subjects without these abnormalities $(\mathrm{P}>0.05)$.

\section{Discussion}

The present study was designed to further investigate the association between plasma sCD36 and cardiovascular risk factors among middle-aged type 2 diabetic patients who fulfilled the IDF definition of MetS in Jordan. The current study did not show any significant difference in plasma SCD36 concentrations between the MetS group and age- and gender-matched healthy controls. This finding was inconsistent with results reported in the Handberg et al (14) study in Denmark and in our previous study, which was conducted on a Caucasian sample from Australia (17). In the two studies, plasma sCD36 concentrations were significantly elevated in obese patients with type 2 DM suggesting that plasma sCD36 could act as a marker for type $2 \mathrm{DM}$, insulin resistance and MetS. However, the Danish study cohort further exhibited a significant difference in plasma SCD36 levels between lean and obese control groups (14) that was not replicated among the Australian participants (17). This suggests that differences in plasma sCD36 concentrations may be expected in different study groups from different populations.

Additionally, absolute sCD36 concentrations reported in the present study were considerably lower than those reported in the majority of other studies $(17,22-24)$. Notable exceptions have been reported by Himoto et al (25) and García-Monzón et al (26) who identified even lower sCD36 concentrations compared to those from the current study (Table IV). This variation could be population-specific as cellular CD36 expression may be associated with the inheritance of specific CD36 gene polymorphisms $(27,28)$. Additionally, cellular CD36 expression can be affected by other factors including fat intake (29) and blood glucose levels (30). However, the lack of a widely reliable standardized method for analyzing circulating sCD36 may represent another cause for inconsistent sCD36 concentrations (24). As shown in Table IV, circulating sCD36 was measured by either in-house ELISA or using various commercially available ELISA kits produced by different companies. Assuming that all the available sCD36 kits have similar detection limits, plasma samples that are contaminated with platelets will exhibit larger CD36 concentrations as CD36 is highly expressed on platelets (31). Thus, the use of different centrifugation conditions to prepare 
Table III. Plasma sCD36 concentrations according to certain metabolic variables.

\begin{tabular}{lll}
\hline Variables (n) & Ln sCD36 & P-value $^{\mathrm{a}}$ \\
\hline
\end{tabular}

Body mass index, $\mathrm{kg} / \mathrm{m}^{2}$

$<30$ (24)

$\geq 30$ (53)

$7.88 \pm 0.79$

$8.14 \pm 0.86$

Fasting glucose, $\mathrm{mmol} / \mathrm{l}$

$\leq 6.1(40)$

$>6.1$ (37)

$7.88 \pm 0.84$

0.06

$8.25 \pm 0.82$

Total cholesterol, $\mathrm{mmol} / \mathrm{l}$

$<5(52)$

$\geq 5(25)$

$8.08 \pm 0.08$

$8.01 \pm 0.87$

High-density lipoprotein, $\mathrm{mmol} / \mathrm{l}$

$\leq 0.9$ (32)

$>0.9$ (45)

$8.27 \pm 0.75$

0.63

$7.90 \pm 0.88$

Low-density lipoprotein, $\mathrm{mmol} / \mathrm{l}$

$<3(42)$

$\geq 3$ (35)

$8.01 \pm 0.84$

$8.11 \pm 0.85$

Triglycerides, $\mathrm{mmol} / \mathrm{l}$

$<1.7$ (46)

$\geq 1.7$ (31)

$8.08 \pm 0.82$

0.76

$8.02 \pm 0.90$

Hypertension

Yes (22)

No (55)

$8.35 \pm 0.83$

0.06

$7.94 \pm 0.83$

Cardiovascular disease

\begin{tabular}{lll} 
Yes (13) & $8.39 \pm 0.97$ & 0.12 \\
No (64) & $7.99 \pm 0.81$ & \\
\hline
\end{tabular}

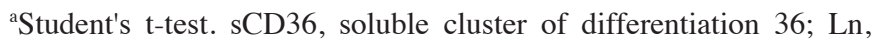
natural logarithm.

plasma prior to CD36 analysis would be expected to impact the amount of residual platelets and hence affect the consistency of sCD36 concentration measurements.

Results from the present study have also shown a lack of correlation between plasma SCD36 concentrations and biochemical parameters that determine the traditional cardiovascular risk factors including BMI, waist circumference, FBG, total cholesterol, HDL-cholesterol, LDL-cholesterol and triglycerides (Table II). In addition, sCD36 concentrations were not correlated with kidney function biochemical parameters and blood parameters, as shown in Table II. Further multiple regression analysis did not show any significant independent predictor of plasma $\mathrm{SCD} 36$ concentrations among all the parameters shown in Table II. This result confirms that plasma sCD36, which was not significantly elevated in the MetS group, is not associated with the traditional cardiovascular risk factors among middle-aged subjects (53.8 \pm 9.7 years). Conversely, in healthy young subjects (18-25 years old) (22), plasma sCD36 concentrations were significantly elevated in obese subjects compared to normal weight subjects and SCD36 was significantly correlated with obesity and LDL-cholesterol. Similarly, in early onset coronary artery disease patients (24),
sCD36 was negatively correlated with BMI, waist circumference, $\mathrm{RBC}$ count, $\mathrm{Hgb}$, glucose and systolic blood pressure, and it was positively correlated with age and HDL-cholesterol. These results (24) suggested an association between sCD36 concentrations and components of MetS including hyperglycemia, hypertension, dyslipidemia and obesity. The correlation of the sCD36 levels with plasma triglycerides was also reported by Knøsgaard et al (32), and triglycerides were found to be significant independent predictors of sCD36 concentrations in morbidly obese subjects. In a nondiabetic healthy population, sCD36 was also significantly correlated with triglycerides, insulin and insulin sensitivity (33). However, the present study was not able to confirm any association between plasma sCD36 concentrations and cardiovascular risk factors, patient age, type 2 DM duration, kidney function parameters and blood cell counts.

To further investigate whether plasma sCD36 concentrations are significantly different according to individual metabolic abnormalities, patients and controls were subgrouped as shown in Table III. The sCD36 concentrations were not significantly different in subjects with obesity, fasting hyperglycemia, hypercholesterolemia, low HDL-cholesterol, high LDL-cholesterol, hypertriglyceridemia, hypertension or coronary vascular disease compared to those without these abnormalities. This confirms that SCD36 is not associated with these metabolic abnormalities in the present study group. Similar results were reported in the study by Ramos-Arellano et al (22) except that subjects with hypertriglyceridemia or hypertension had higher sCD36 concentrations than subjects without these abnormalities.

Taken together and based on the measurement of absolute sCD36 concentrations by ELISA, the present study suggests that $\mathrm{SCD} 36$ was not associated with cardiovascular risk factors among patients with MetS and its concentrations were not significantly different compared to healthy controls. This result is inconsistent with what has been reported previously as discussed; however, it may represent a unique result for the present study population. However, this research has certain limitations that may influence its results. For example, results from different commercially available human sCD36 ELISA kits were not examined due to fund limitations. This would allow a generally reliable standardized method for determining sCD36 concentrations. Furthermore, we have previously shown that sCD36 is not truly soluble but is entirely associated with plasma microparticles (MPs) that shed from cell membranes as a result of cell activation or cell apoptosis (34). Additionally, the plasma levels of CD36-positive MPs and sCD36 concentrations were strongly correlated together (17). However, the CD36-positive MPs could not be measured in the current study as the required equipment for such analysis was not available. Thus, the use of a standardized ELISA that could be performed in any basic laboratory over the specialized flow cytometry methods required to enumerate MPs would be favorable to detect sCD36 if it were to be used as a biomarker.

However, further studies are required to determine CD36 tissue expression in the current study population. Such information is required to justify why plasma sCD36 concentrations were low and to check whether sCD36 concentrations do reflect its tissue expression. In addition, further research should be performed to establish a well-standardized method for analyzing circulating CD36 including plasma preparation 


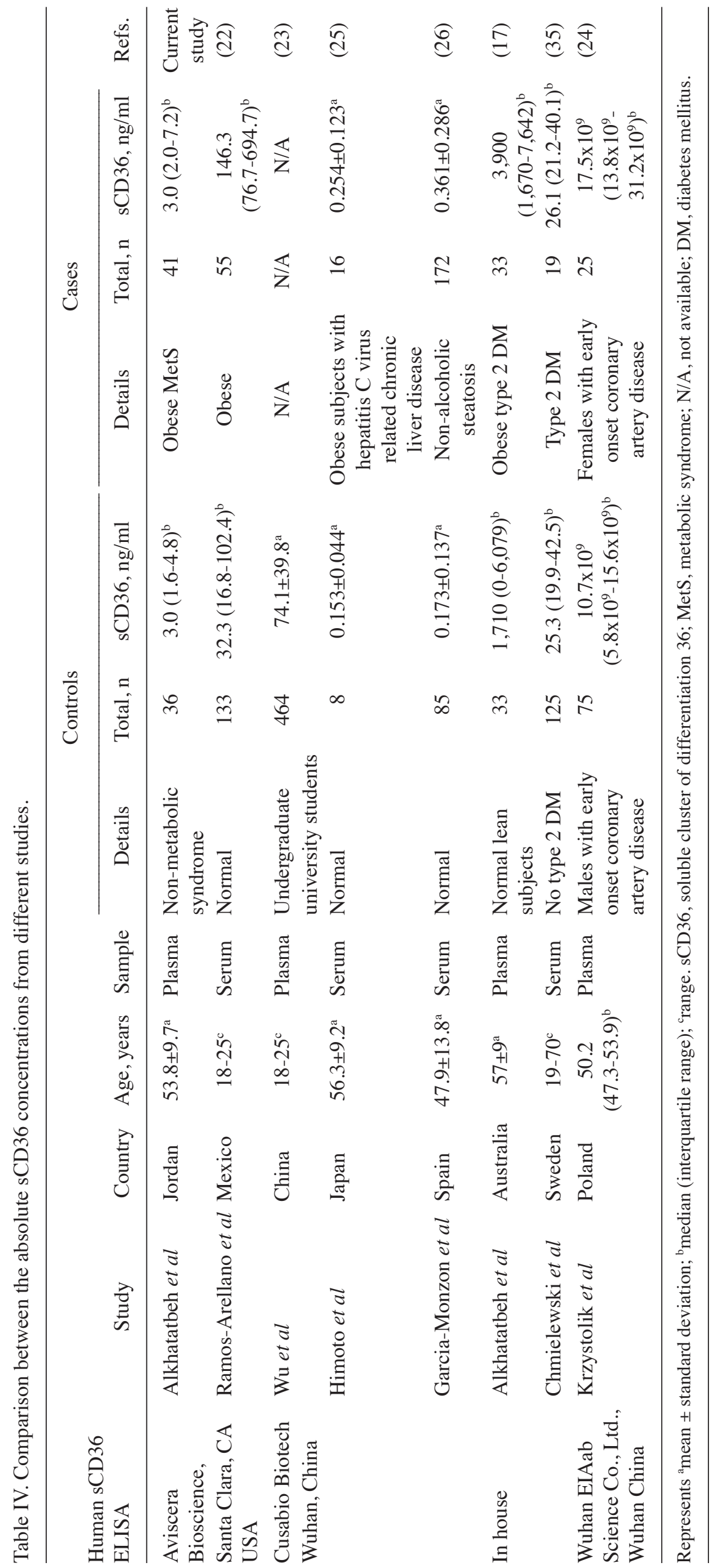


to produce comparable sCD36 results between different study groups. Additional studies are also required to assess differences in sCD36 levels among different populations and to check if population-specific factors may affect sCD36 concentrations.

\section{Acknowledgements}

The authors would like to thank the Deanship of Research at Jordan University of Science and Technology for providing the financial support for the present study.

\section{References}

1. Finegold JA, Asaria P and Francis DP: Mortality from ischaemic heart disease by country, region, and age: Statistics from World Health Organisation and United Nations. Int J Cardiol 168: 934-945, 2013.

2. Tabei SM, Senemar S, Saffari B, Ahmadi Z and Haqparast S: Non-modifiable factors of coronary artery stenosis in late onset patients with coronary artery disease in Southern Iranian population. J Cardiovasc Thorac Res 6: 51-55, 2014.

3. Cannon CP: Cardiovascular disease and modifiable cardiometabolic risk factors. Clin Cornerstone 8: 11-28, 2007.

4. Grundy SM: Metabolic syndrome: A multiplex cardiovascular risk factor. J Clin Endocrinol Metab 92: 399-404, 2007.

5. Consultation WHO: Definition, diagnosis and classification of diabetes mellitus and its complications. World Health Organization, Geneva, Switzerland, pp31-33, 1999.

6. Balkau B and Charles MA; European Group for the Study of Insulin Resistance (EGIR): Comment on the provisional report from the WHO consultation. Diabet Med 16: 442-443, 1999.

7. Expert Panel on Detection, Evaluation, and Treatment of High Blood Cholesterol in Adults: Executive Summary of The Third Report of The National Cholesterol Education Program (NCEP) Expert Panel on Detection, Evaluation, And Treatment of High Blood Cholesterol In Adults (Adult Treatment Panel III) JAMA 285: 2486-2497, 2001.

8. International Diabetes Federation: The IDF Consensus Worldwide Definition of the Metabolic Syndrome. International Diabetes Federation, Brussels, Belgium, 2006.

9. Ge Y and Wang TJ: Identifying novel biomarkers for cardiovascular disease risk prediction. J Intern Med 272: 430-439, 2012

10. Khot UN, Khot MB, Bajzer CT, Sapp SK, Ohman EM, Brener SJ, Ellis SG, Lincoff AM and Topol EJ: Prevalence of conventional risk factors in patients with coronary heart disease. JAMA 290: 898-904, 2003.

11. Montgomery JE and Brown JR: Metabolic biomarkers for predicting cardiovascular disease. Vasc Health Risk Manag 9 $37-45,2013$

12. van Holten TC, Waanders LF, de Groot PG, Vissers J, Hoefer IE, Pasterkamp G, Prins MW and Roest M: Circulating biomarkers for predicting cardiovascular disease risk; a systematic review and comprehensive overview of meta-analyses. PLoS One 8 e62080, 2013.

13. Libby P, Geng YJ, Aikawa M, Schoenbeck U, Mach F, Clinton SK, Sukhova GK and Lee RT: Macrophages and atherosclerotic plaque stability. Curr Opin Lipidol 7: 330-335, 1996.

14. Handberg A, Levin $K$, Højlund $K$ and Beck-Nielsen $H$ : Identification of the oxidized low-density lipoprotein scavenger receptor CD36 in plasma: A novel marker of insulin resistance. Circulation 114: 1169-1176, 2006.

15. Park YM: CD36, a scavenger receptor implicated in atherosclerosis. Exp Mol Med 46: e99, 2014.

16. Bonen A, Campbell SE, Benton CR, Chabowski A, Coort SL, Han XX, Koonen DP, Glatz JF and Luiken JJ: Regulation of fatty acid transport by fatty acid translocase/CD36. Proc Nutr Soc 63: 245-249, 2004

17. Alkhatatbeh MJ, Enjeti AK, Acharya S, Thorne RF and Lincz LF: The origin of circulating CD36 in type 2 diabetes. Nutr Diabetes 3: e59, 2013.

18. Glintborg D, Højlund K, Andersen M, Henriksen JE, Beck-Nielsen H and Handberg A: Soluble CD36 and risk markers of insulin resistance and atherosclerosis are elevated in polycystic ovary syndrome and significantly reduced during pioglitazone treatment. Diabetes Care 31: 328-334, 2008.
19. Handberg A, Skjelland M, Michelsen AE, Sagen EL, Krohg-Sørensen K, Russell D, Dahl A, Ueland T, Oie E, Aukrust $\mathrm{P}$, et al: Soluble CD36 in plasma is increased in patients with symptomatic atherosclerotic carotid plaques and is related to plaque instability. Stroke 39: 3092-3095, 2008.

20. Khader Y, Batieha A, Jaddou H, El-Khateeb M and Ajlouni K: Metabolic syndrome and its individual components among Jordanian children and adolescents. Int J Pediatr Endocrinol 2010: 316170, 2010.

21. American Diabetes Association: Diagnosis and classification of diabetes mellitus. Diabetes Care 33 (Suppl 1): S62-S69, 2010

22. Ramos-Arellano LE, Muñoz-Valle JF, De la Cruz-Mosso U, Salgado-Bernabé AB, Castro-Alarcón N and Parra-Rojas I: Circulating CD36 and oxLDL levels are associated with cardiovascular risk factors in young subjects. BMC Cardiovasc Disord 14: 54, 2014.

23. Wu S, Yang D, Wei H, Wang B, Huang J, Li H, Shima M, Deng F and Guo X: Association of chemical constituents and pollution sources of ambient fine particulate air pollution and biomarkers of oxidative stress associated with atherosclerosis: A panel study among young adults in Beijing, China. Chemosphere 135: 347-353, 2015.

24. Krzystolik A, Dziedziejko V, Safranow K, Kurzawski G, Rać M, Sagasz-Tysiewicz D, Poncyljusz W, Jakubowska K, Chlubek D and Rać ME: Is plasma soluble CD36 associated with cardiovascular risk factors in early onset coronary artery disease patients? Scand J Clin Lab Invest 75: 398-406, 2015.

25. Himoto T, Tani J, Miyoshi H, Morishita A, Yoneyama H, Kurokohchi K, Inukai M, Masugata $\mathrm{H}$, Goda F, Senda S, et al: Investigation of the factors associated with circulating soluble CD36 levels in patients with HCV-related chronic liver disease. Diabetol Metab Syndr 5: 51, 2013.

26. García-Monzón C, Lo Iacono O, Crespo J, Romero-Gómez M, García-Samaniego J, Fernández-Bermejo M, Domínguez-Díez A, Rodríguez de Cía J, Sáez A, Porrero JL, et al: Increased soluble CD36 is linked to advanced steatosis in nonalcoholic fatty liver disease. Eur J Clin Invest 44: 65-73, 2014.

27. Masuda Y, Tamura S, Matsuno K, Nagasawa A, Hayasaka K, Shimizu C and Moriyama T: Diverse CD36 expression among Japanese population: Defective CD36 mutations cause platelet and monocyte CD36 reductions in not only deficient but also normal phenotype subjects. Thromb Res 135: 951-957, 2015.

28. Ghosh A, Murugesan G, Chen K, Zhang L, Wang Q, Febbraio M, Anselmo RM, Marchant K, Barnard J and Silverstein RL: Platelet CD36 surface expression levels affect functional responses to oxidized LDL and are associated with inheritance of specific genetic polymorphisms. Blood 117: 6355-6366, 2011.

29. Alexander Aguilera A, Hernández Díaz G, Lara Barcelata M, Angulo Guerrero O and Oliart Ros RM: Induction of Cd36 expression elicited by fish oil PUFA in spontaneously hypertensive rats. J Nutr Biochem 17: 760-765, 2006.

30. Griffin E, Re A, Hamel N, Fu C, Bush H, McCaffrey T and Asch AS: A link between diabetes and atherosclerosis: Glucose regulates expression of CD36 at the level of translation. Nat Med 7: 840-846, 2001.

31. Tandon NN, Kralisz U and Jamieson GA: Identification of glycoprotein IV (CD36) as a primary receptor for platelet-collagen adhesion. J Biol Chem 264: 7576-7583, 1989.

32. Knøsgaard L, Thomsen SB, Støckel M, Vestergaard H and Handberg A: Circulating sCD36 is associated with unhealthy fat distribution and elevated circulating triglycerides in morbidly obese individuals. Nutr Diabetes 4: e114, 2014.

33. Handberg A, Højlund K, Gastaldelli A, Flyvbjerg A, Dekker JM, Petrie J, Piatti P and Beck-Nielsen H; RISC Investigators: Plasma sCD36 is associated with markers of atherosclerosis, insulin resistance and fatty liver in a nondiabetic healthy population. J Intern Med 271: 294-304, 2012.

34. Alkhatatbeh MJ, Mhaidat NM, Enjeti AK, Lincz LF and Thorne RF: The putative diabetic plasma marker, soluble CD36, is non-cleaved, non-soluble and entirely associated with microparticles. J Thromb Haemost 9: 844-851, 2011.

35. Chmielewski M, Bragfors-Helin AC, Stenvinkel P, Lindholm B and Anderstam B: Serum soluble CD36, assessed by a novel monoclonal antibody-based sandwich ELISA, predicts cardiovascular mortality in dialysis patients. Clin Chim Acta 411: 2079-1082, 2010 